\title{
Seeing past the appendix: the role of ultrasound in right iliac fossa pain
}

\author{
White EK, MacDonald L, Johnson G and Rudralingham V \\ University Hospitals of South Manchester, Wythenshawe Hospital, Manchester, UK \\ Corresponding author: White EK. Email: emilykwhite@doctors.org.uk
}

\begin{abstract}
Acute right iliac fossa pain is a common surgical presentation. The presentation is often non-specific and encompasses a wide differential, which creates a diagnostic challenge. Ultrasound is commonly the initial cross-sectional imaging modality and can be used as a tool to triage patients appropriately; assessing for appendicitis and other salient findings, which may indicate an alternative condition. Additionally, the dynamic nature of this imaging modality enables patient interaction. Following a systematic assessment of the abdomen and pelvis, a more focused interrogation of the right iliac fossa is performed. In this pictorial review, we illustrate the sonographic features of appendicitis and other conditions that can mimic appendicitis in its presentation. This highlights that through a systematic approach, it is possible to distinguish between these different pathologies, enabling clinicians to optimally manage the patient.
\end{abstract}

Keywords: Ultrasound, emergency radiology, appendix

Ultrasound 2014; 22: 104-112. DOI: 10.1177/1742271X13514385

\section{Introduction}

The ultimate aim of ultrasound in the acute setting for the assessment of patients with acute right iliac fossa pain, is to detect the presence of acute appendicitis, which will require prompt surgery, or to provide a robust alternative explanation for the patient's symptoms. In some instances, this may involve further alternative cross-sectional imaging with CT and/or MRI to confirm findings.

The success of ultrasound is operator-dependent and is reliant on thorough assessment of the entire abdominal cavity. In cases of acute right iliac fossa pain, most patients are assessed in a fasting state. Initial assessment is performed with a curvilinear $(5-7 \mathrm{MHz})$ probe to look at the solid organs, gallbladder, biliary tree and the pelvic viscera.

Once a general survey has been carried out, a more targeted assessment of the right iliac fossa can be performed. The bowel is usually interrogated with both a curvilinear and a high-resolution linear probe (9-12 MHz). In our practice, we start in the right upper quadrant with the curvilinear probe, and trace the colon down from the hepatic flexure to the caecal pole, using graded compression sonography. The linear probe should be routinely used to scrutinise suspicious regions. Doppler assessment may also add information regarding vascularity and nature of abnormality.

When normal bowel is compressed, the intra-luminal gas is displaced away and peristaltic activity can be visualised. In contrast, abnormal bowel has a striking appearance showing no peristalsis and does not alter with compression.
This technique also allows for clinical judgement of true peritoneal irritation or rebound tenderness. In addition, being able to scan at the point of maximum tenderness, as indicated by the patient, provides an index of clinical suspicion and justifies the urgency for further cross-sectional imaging when findings are equivocal on ultrasound.

In women, transvaginal scanning is invaluable and should be considered mandatory to inspect the uterus and adnexal structures.

In this paper, through a series of cases, we present the typical sonographic appearances associated with acute appendicitis, as well as those appearances associated with a number of other pathological conditions which may mimic appendicitis in clinical presentation.

\section{Appendicitis}

The normal appendix is a thin-walled, compressible blindending tubular structure, arising from the posteromedial aspect of the caecum where the taenia coli converge. It usually measures $4 \mathrm{~mm}$ in diameter and $8 \mathrm{~cm}$ in length. Inflammation of the appendix occurs secondary to obstruction of the appendiceal lumen. Continued mucous secretion causes dilation of the appendix and increased intraluminal pressure, compromising venous return and causing eventual necrosis. ${ }^{1}$

The classical history for appendicitis is a patient presenting with central umbilical pain that moves to the right iliac fossa. On examination, the patient has signs of localised 
peritonism in the right iliac fossa. Blood tests often demonstrate a mildly raised white cell count and CRP.

Ultrasound examination has been found to be most specific if performed using the graded compression technique, which involves applying slowly increasing pressure with a high-frequency transducer over the region of maximal tenderness (Figure 1). ${ }^{2}$ The majority of women of child-bearing age, who present with suspected acute appendicitis, benefit from pre-operative ultrasound, with a statistically significant lower negative appendicectomy rate than those women who did not undergo imaging. ${ }^{3}$

The sonographic features of acute appendicitis are visualisation of an aperistaltic, thick-walled, blind-ending, tubular structure with a diameter $>6 \mathrm{~mm}$ with compression. The presence of an appendicolith with posterior acoustic shadowing (Figure 2) is a specific feature but not seen in all cases. However, the only sonographic sign may be echogenic fat within the right iliac fossa (Figure 3 ). This is non-specific and indicates inflammation in the right iliac fossa. If this is found in isolation, then further imaging with either CT or MRI is advised to further assess. If the appendix has perforated, a pericaecal fluid collection or even a discontinuity in the wall of the appendix may be demonstrated.

Patients with acute appendicitis are managed surgically with either an open or laparoscopic appendicectomy.

\section{Gastrointestinal}

\section{Crohn's disease: terminal ileitis}

Crohn's disease is a chronic inflammatory disorder of the gastrointestinal tract. It classically affects Caucasians and has no gender predominance. The terminal ileum and colon are most commonly affected, but any part of the gastrointestinal tract may be involved. The disease is characterised by full thickness bowel wall inflammation with aphthous ulcers, deep fissures and luminal narrowing.

Ultrasound has an important role in the imaging of both acute exacerbations and long-term follow-up of the disease. Ultrasound is a sensitive, non-invasive imaging modality which does not involve the use of ionising radiation.

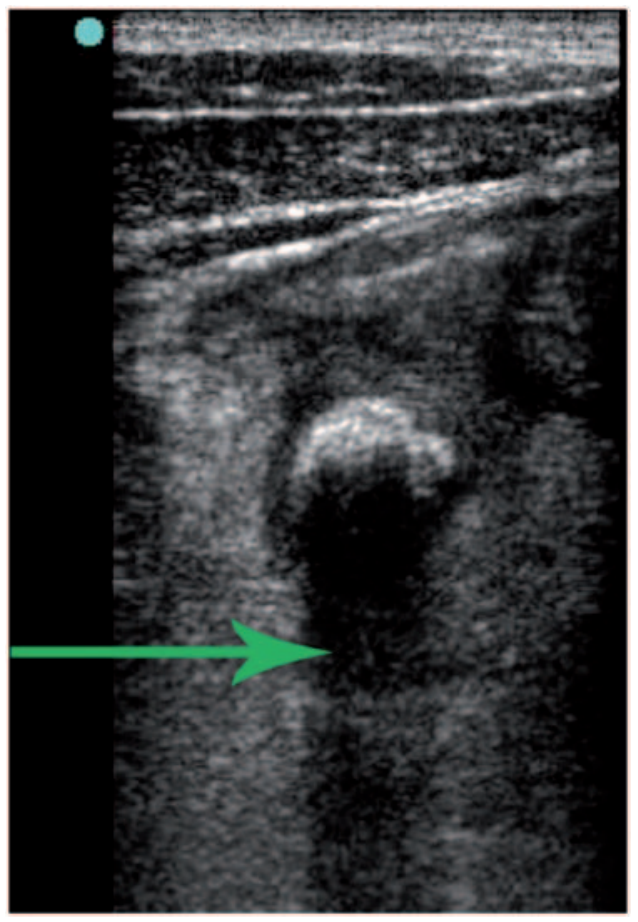

Figure 2 Appendicolith with acoustic shadowing [arrow]

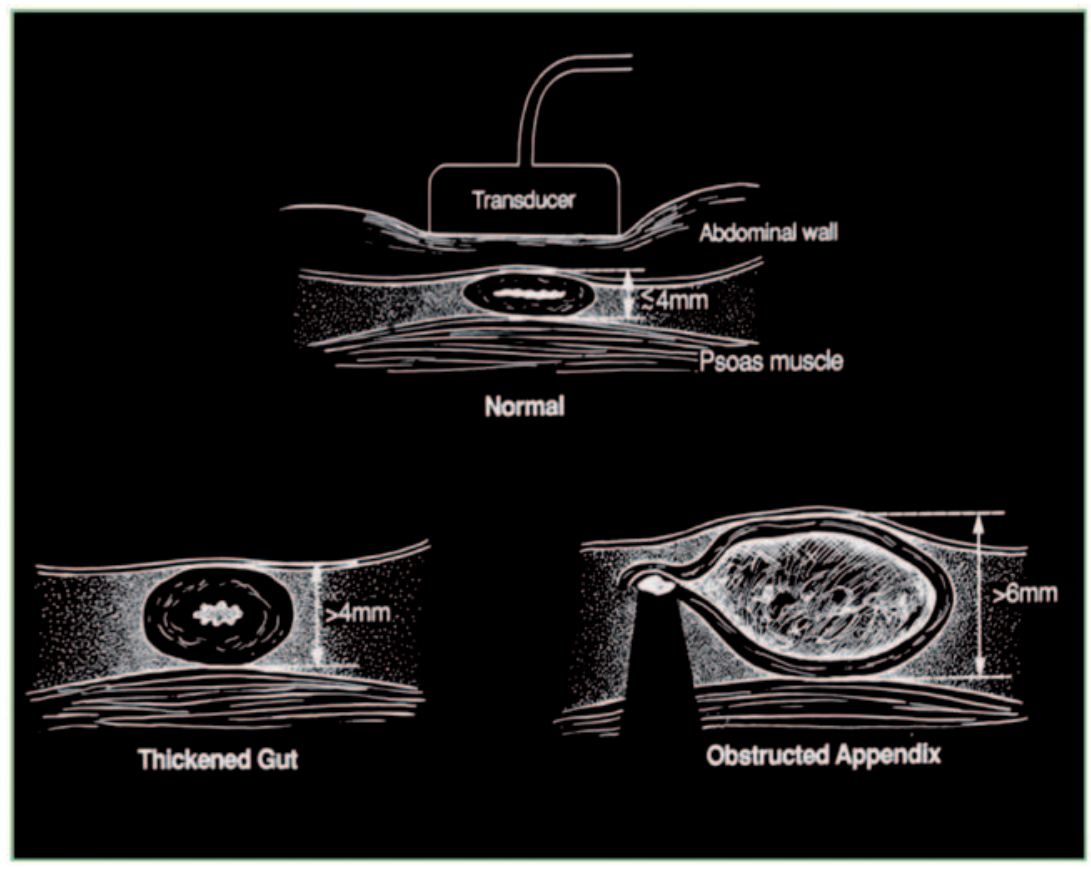

Figure 1 Pictorial representation of graded compression sonography 
This is particularly relevant in the young populations frequently affected.

Sonographic features of acute Crohn's disease include concentric mural thickening involving a long segment of terminal ileum with decreased peristalsis. The wall echogenicity varies dependent on the activity of disease. Typically, there is exaggeration of the stratified layers of the gut wall. Echogenic inflamed fat may be seen 'creeping' over the wall (Figure 4) with adjacent separation of bowel loops, and enlarged mesenteric nodes. Hyperaemia may be observed on Doppler (Figure 5) in active disease and careful assessment should be made to look for evidence of localised complications such as fistula, abscess or obstruction.

The management of patients with Crohn's disease is complex, however initial management is often medical. Therefore, recognising this condition on ultrasound is important as it avoids unnecessary surgery. There is overlap of the ultrasound features with other forms of acute ileitis typically from infective causes such as Yersinia and

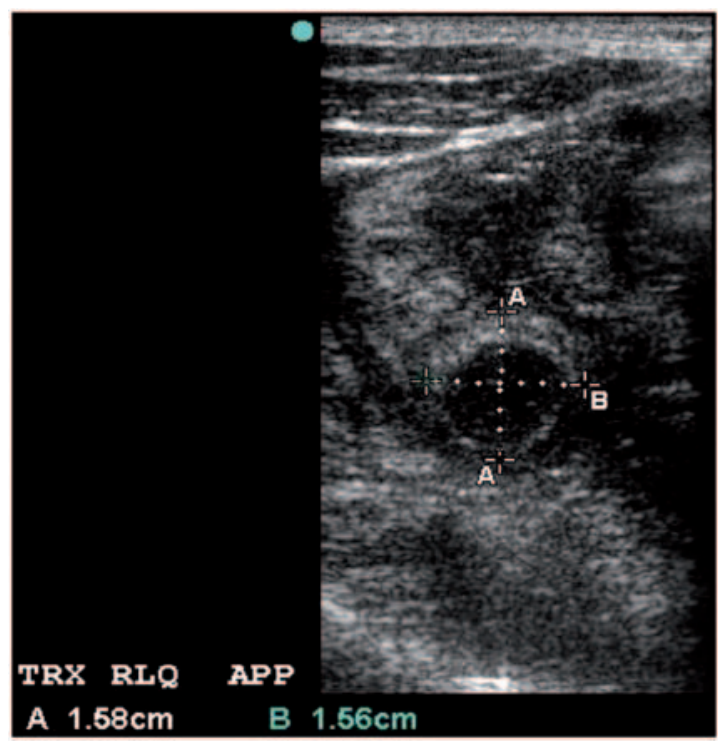

Figure 3 Echogenic fat as seen in acute appendicitis

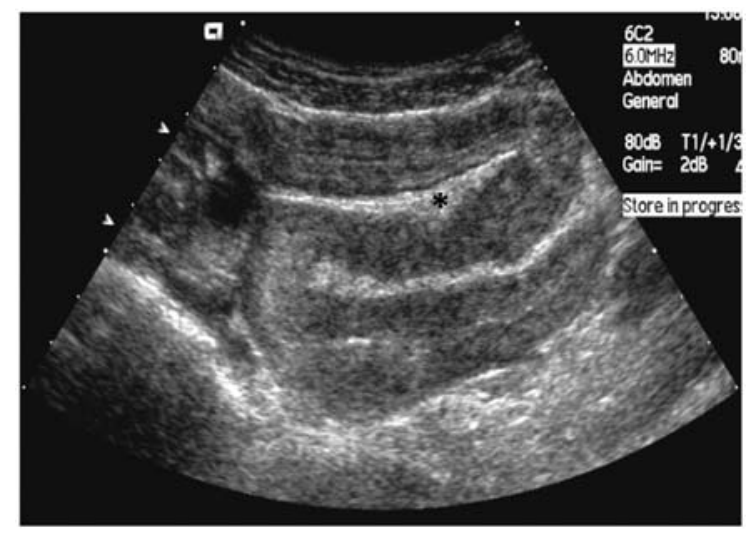

Figure 4 Long segment of mural thickening seen in longitudinal section with echogenic 'creeping' fat $\left.{ }^{\star}\right]$ as seen in Crohn's disease
Campylobacter, ${ }^{4}$ hence the importance of good clinical correlation with the sonographic findings.

\section{Right-sided diverticulitis}

Diverticulosis is the presence of diverticula and diverticulitis is the inflammation of a diverticula. Although diverticulosis is most often confined to the descending and sigmoid colon, the ascending colon can also be affected. In left-sided diverticular disease, the diverticula are usually pseudodiverticula, consisting of mucosa and sub-mucosa alone, whereas right-sided diverticula tend to be true diverticula, containing all colonic layers. Right-sided disease is known to affect a younger population. ${ }^{5,6}$ The aetiology of true right-sided diverticular disease is uncertain.

In acute right-sided diverticulitis, the clinical presentation is usually indistinguishable from acute appendicitis. On ultrasound, the inflamed diverticulum is seen as an out-pouching arising from the right colon, containing either fluid or a faecolith, surrounded by echogenic inflamed fat (Figure 6). Some studies have shown ultrasound to have a similar diagnostic accuracy to CT imaging. ${ }^{7}$ Recognising this condition is crucial as this can usually be

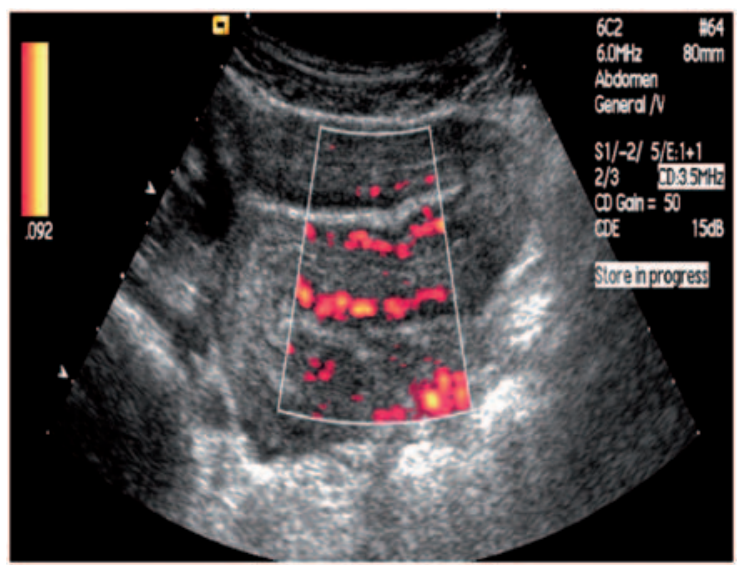

Figure 5 Increased Doppler flow demonstrating hyperaemia as seen in active Crohn's disease

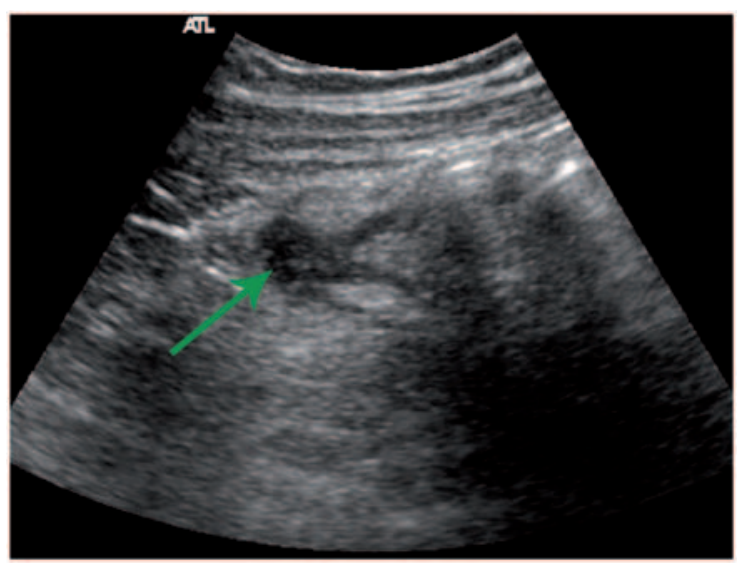

Figure 6 Focal outpouching [arrow] representing left colonic diverticulum 
managed conservatively with intravenous antibiotics and settles without surgery.

\section{Caecal tumour}

In the older patient population, who present with acute right iliac fossa pain, an important diagnosis to consider is an underlying obstructing caecal tumour at the base of the appendix, presenting as acute appendicitis. Alternatively, the caecal tumour may itself be the primary diagnosis due to localised tumour perforation or peritoneal infiltration.

On ultrasound, neoplastic thickening usually involves a short segment of bowel with disruption of the gut wall layers. The bowel wall typically appears solid and hypoechoic (Figure 7). If a solid mass is identified within the right colon on ultrasound, then CT is recommended to further assess (Figure 8). CT will provide more information on the nature of thickening, stage of the tumour and help determine optimum management for the patient.

\section{Intussusception}

Intussusception is the telescopic invagination of a segment of bowel, intussusceptum, into a segment of more distal bowel, intussuscipiens. ${ }^{8}$ This most commonly occurs in

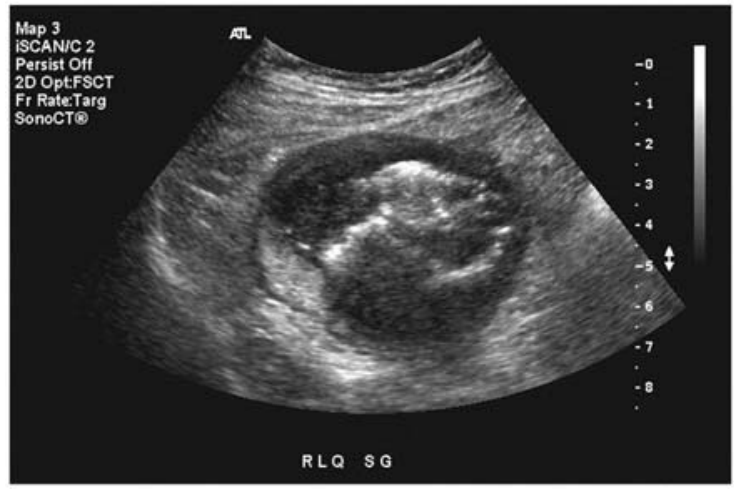

Figure 7 Axial ultrasound image of colon cancer demonstrating asymmetric hypoechoic bowel wall thickening with disruption of the gut wall layers the paediatric population, with $40 \%$ of cases affecting those aged 3-6 months, although it can also occur in adults. In children aged 3-6 months, intussusception is commonly idiopathic with no discrete pathological lead point identified, whereas in adults, pathology acting as a lead point needs to be considered. Presenting features include pain and vomiting (90\%) and in $60 \%$ of paediatric cases blood per rectum ('redcurrant stool'). ${ }^{9}$ A palpable mass may also be felt.

Although an intussusception can occur at any point in the ileum or colon, the most common type in children is ileocolic $(>75 \%)$, where a segment of terminal ileum invaginates into the caecum. A prompt diagnosis is particularly important to make in a paediatric patient, as the aim is to avoid vascular compromise of the bowel secondary to strangulation of the mesentery. This is in contrast to adult patients, where the condition can be a transient self-limiting phenomenon. ${ }^{8}$

Ultrasound is the initial investigation of choice, with high sensitivity and specificity of between $98-100 \%$ and $88-100 \%$, respectively. ${ }^{8}$ The classical sonographic finding in the transverse plane is a 'target' or 'doughnut' sign (Figure 9). This is the result of concentric hypoechoic and hyperechoic rings (representing the intussuscipiens), with a central more echogenic portion (representing the inflamed mesentery or the intussusceptum). In the longitudinal plane, the same representation is seen in long section and has been described as a 'sandwich' or 'hayfork' sign. ${ }^{10}$ These sonographic findings are highly specific for intussusception. More non-specific findings include an echogenic mesentery containing lymph nodes. Doppler scanning should always be performed to attempt to ascertain whether there is blood flow in the intussusceptum, as absence may suggest necrosis.

\section{Gynaecological Ovarian torsion}

Ovarian torsion occurs when there is twisting of the vascular pedicle of the ovary, fallopian tube or both. This can result in ischaemia, and ultimately infarction of these structures.

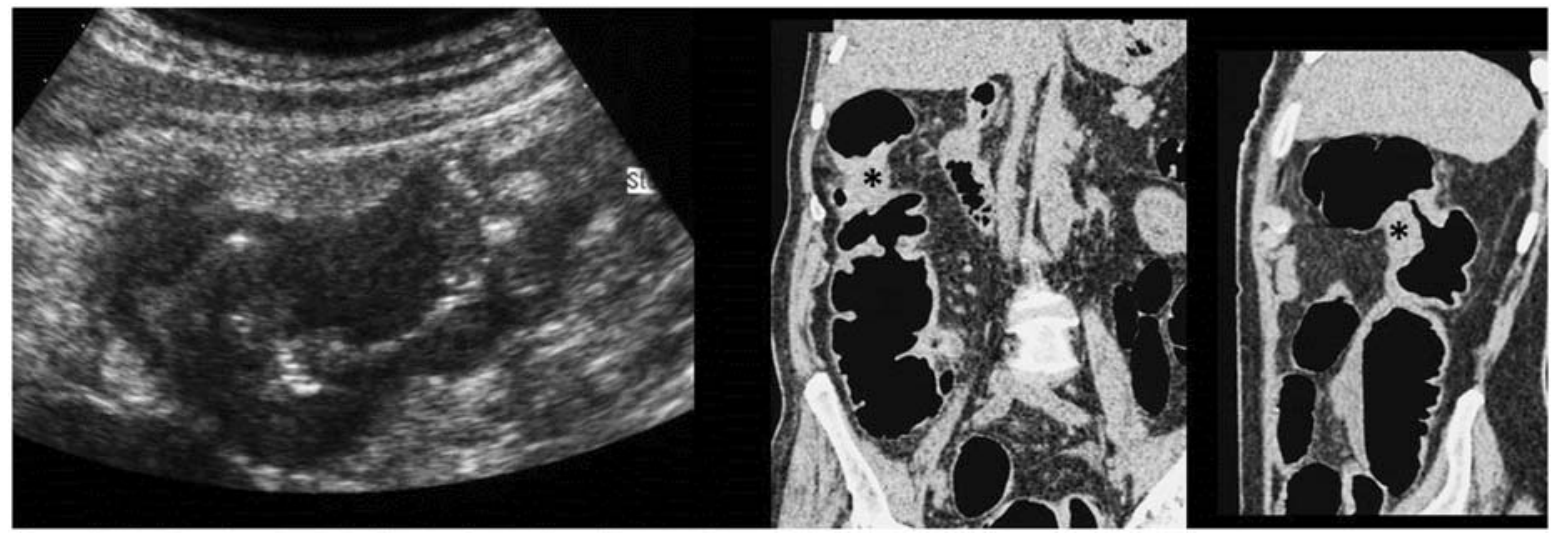

Figure 8 Colonic tumour: sagittal ultrasound image demonstrating a short segment of bowel thickening with disruption of the gut wall layers corresponding to an 'apple core' lesion on coronal and sagittal CT images [*] 
The mean age of incidence is 11 years. In the paediatric population, the most common cause is excessive mobility of the ovary, resulting in torsion of normal adnexal structures. However, this can be associated with an ovarian mass, which acts as a lead point. The mass can be either a functional cyst $(60 \%)$ or a neoplasm $(40 \%)$.

Sonographic features include a unilateral, uniformly enlarged ovary containing multiple small peripheral ovarian follicles measuring 8-12 $\mathrm{mm}$ in diameter, reflecting congestion of the ovary (Figure 10). This sign is specific but not very sensitive. $^{11}$

Non-specific findings include fluid in the pouch of Douglas and thickening of the fallopian tube on the affected side. Colour and pulse wave Doppler assessment is important as absence of Doppler flow within the ovary may be demonstrated in torsion (Figure 11). Typically, there is initial loss of the venous flow, followed by absence of both arterial and venous flow. The whirlpool sign represents the twisted vascular pedicle of the ovary and is a definitive sign of torsion. ${ }^{12}$

Torsion of the ovary is a gynaecological emergency and the patient needs urgent surgery to try and prevent ovarian necrosis.

\section{Haemorrhagic ovarian cyst}

Functional cysts such as follicular cysts and corpus luteum cysts have the potential to develop internal haemorrhage. Although not pathologically sinister, this can be symptomatic causing abdominal pain and require follow-up ultrasound to ensure resolution.

In cases of mimicked appendicitis, typical appearances include a single unilateral cyst of the right ovary with internal echoes but no internal Doppler flow. In the acute stage, a 'fishnet weave' pattern or lacelike reticular pattern is often seen (Figure 12). ${ }^{8}$ Clot may adhere to the cyst wall and this

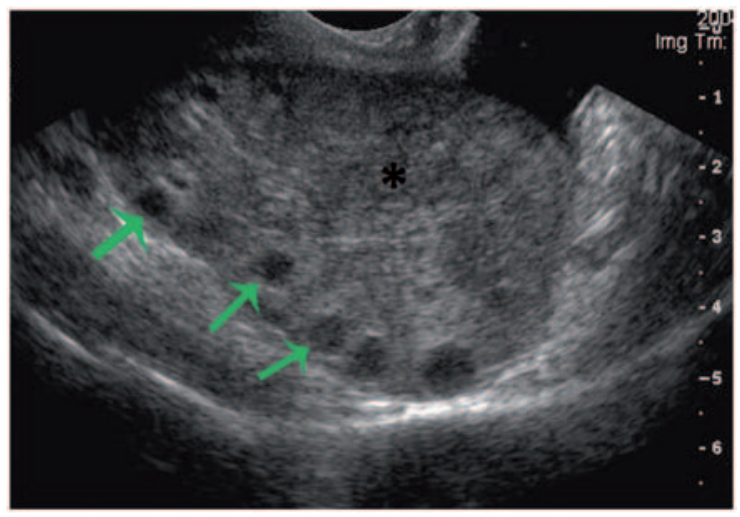

Figure 10 Enlarged ovary [*] with multiple peripheral cysts [arrows] indicative of ovarian congestion, a sensitive sign of torsion

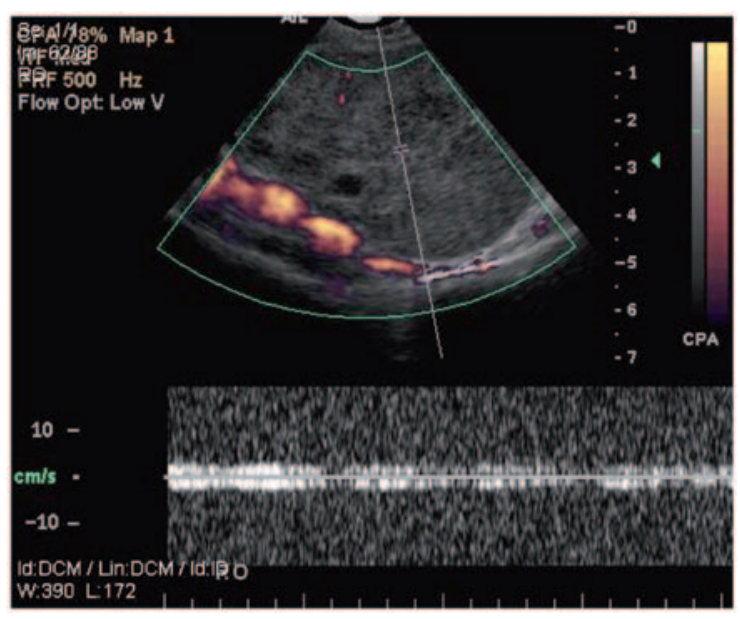

Figure 11 Vascular flow seen within the ovarian pedicle with absent flow within the ovarian stroma

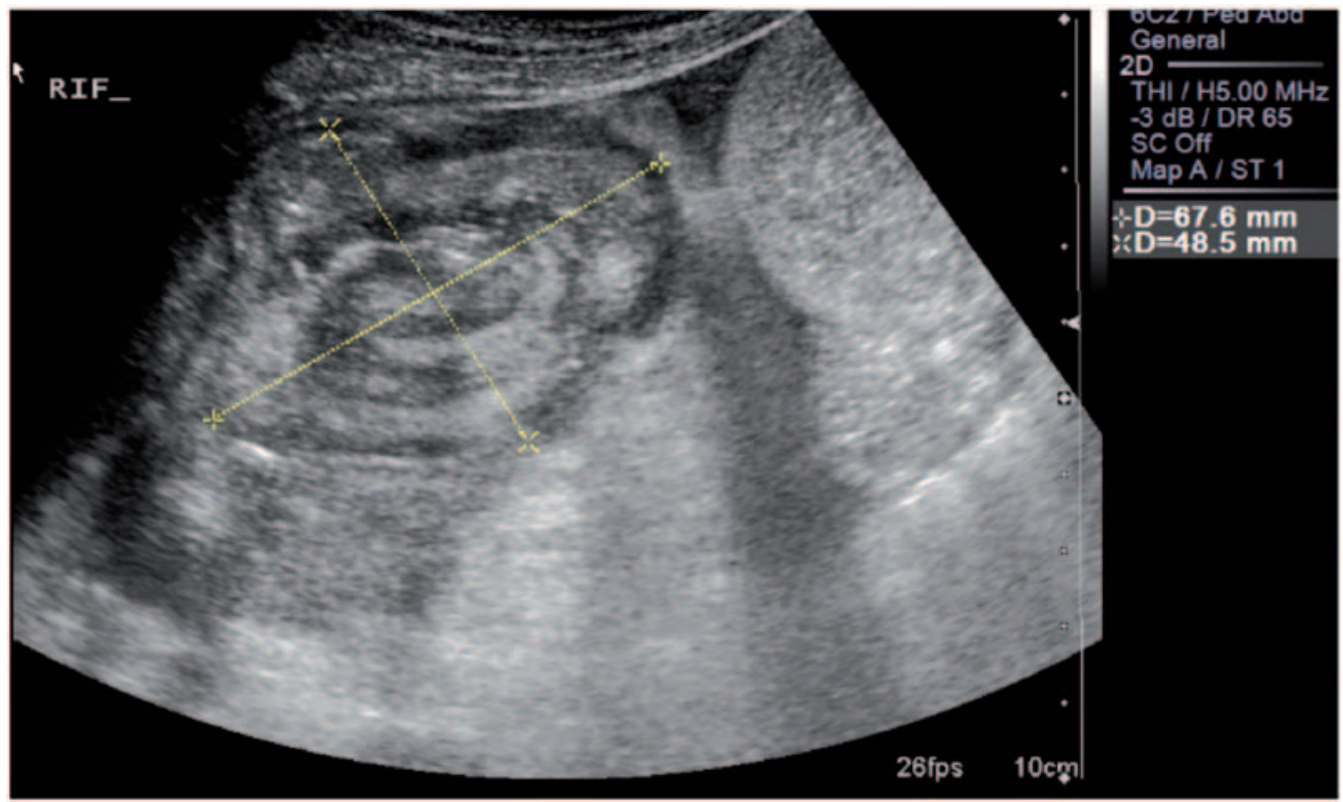

Figure 9 'Target sign': multiple concentric rings as seen in intussusception 


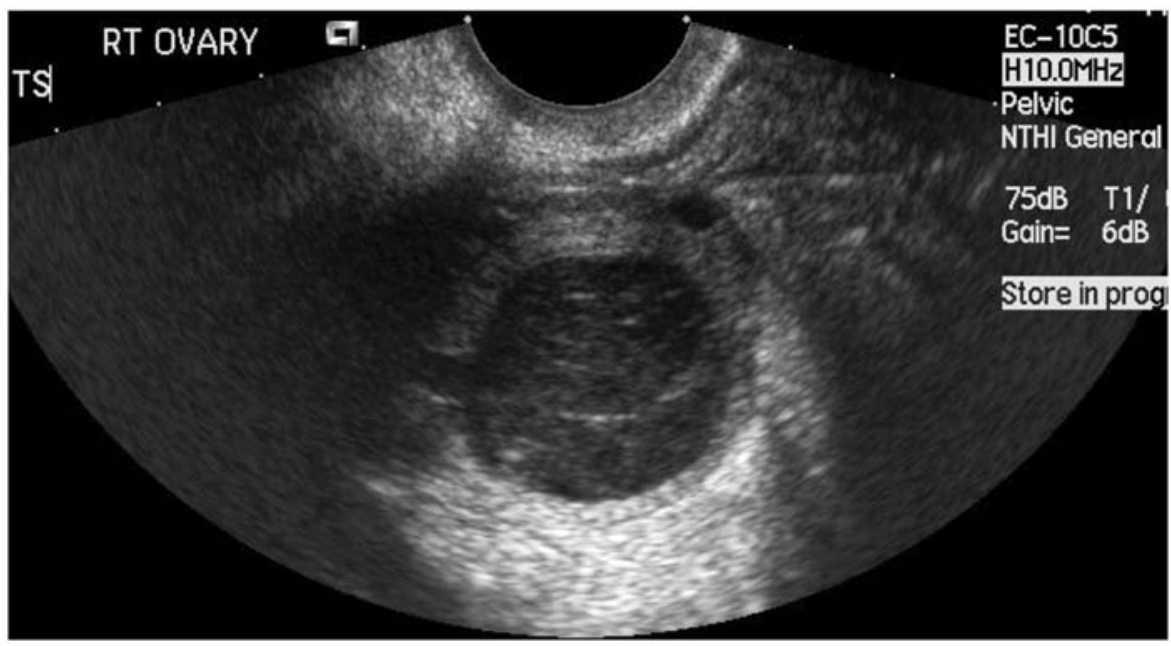

Figure 12 Internal reticular pattern representing haemorrhage within a functional ovarian cyst

can resemble papillary projections seen in neoplastic processes; importantly in a benign haemorrhagic cyst these will not demonstrate blood flow.

Haemorrhagic ovarian cysts usually resolve spontaneously $^{13}$ and a repeat ultrasound scan performed in 6-8 weeks will commonly show involution of the cyst. Premenopausal patients with cysts measuring greater than $5 \mathrm{~cm}$ in maximum diameter and early postmenopausal patients with cysts of any size should be followed up at 6 weeks. ${ }^{14}$ In late postmenopausal women, findings consistent with a haemorrhagic cyst should be considered neoplastic until proven otherwise and should be referred to a gynaecologist for further investigation including Ca125 levels and an MRI to further characterise the lesion.

\section{Ectopic pregnancy $+/$ - rupture}

Ectopic pregnancy is the major cause of pregnancy-related maternal deaths ${ }^{15}$ and is therefore an important differential to consider in any woman of childbearing age presenting with either abdominal pain, an adnexal mass or abnormal bleeding. In these cases, prompt gynaecological opinion and correlation with LMP and human chorionic gonadotrophin (hCG) levels is essential to ensure prompt appropriate management.

The majority of ectopic pregnancies occur in the fallopian tube, but they can also implant in the abdominal cavity, ovary and cervix. A confident sonographic diagnosis of an ectopic pregnancy can be made in $18-26 \%$ of cases; ${ }^{16}$ in these cases a live embryo is identified external to the uterus. In the majority of cases this is not possible and so in the presence of a positive pregnancy test, one of the main aims of ultrasound is to demonstrate the presence or absence of a normal intrauterine pregnancy. Confirmation of an intrauterine pregnancy virtually excludes the diagnosis of an ectopic pregnancy.

There are a number of corroborating findings that can also support the diagnosis of an ectopic pregnancy. These include a complex adnexal mass with the ovary identified separately or a tubal ring sign - an echogenic rim

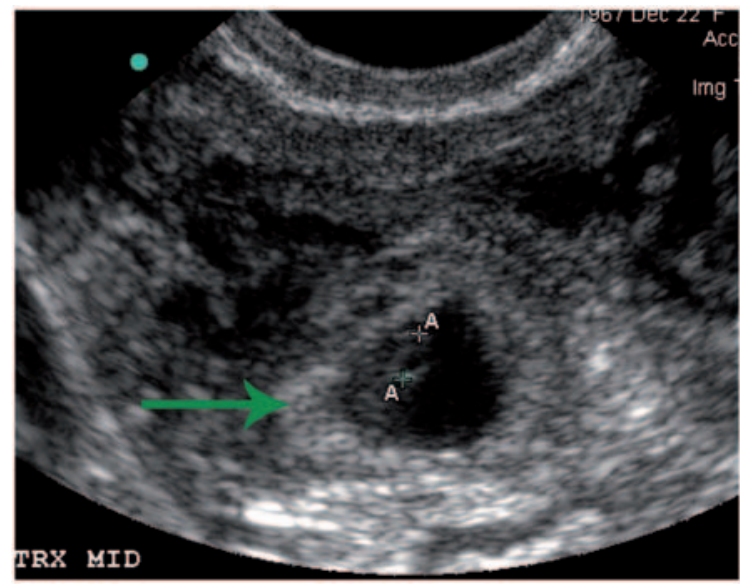

Figure 13 'Tubal ring sign': an echogenic rim [arrow] surrounding an unruptured ectopic pregnancy

surrounding an unruptured ectopic pregnancy with striking hyperaemia on colour Doppler assessment - may be seen. This is commonly referred to as the 'ring of fire' sign (Figure 13). Free fluid in the pelvis is a common finding and can often represent complex haemorrhagic ascites (Figure 14).

An ectopic pregnancy is a gynaecological emergency and ultrasound examination should be performed in conjunction with prompt referral to the gynaecology team.

\section{Pyosalpinx}

A normal fallopian tube measures less than $4 \mathrm{~mm}$ in diameter and is beyond the resolution of standard ultrasound probes, therefore, a visible fallopian tube is always abnormal. In a pyosalpinx, the fallopian tube is filled with pus. Patients present with acute lower abdominal pain as well as elevated inflammatory markers and white cell count. It is often a bilateral finding ${ }^{8}$ which would exclude many of the differential diagnoses already discussed. 


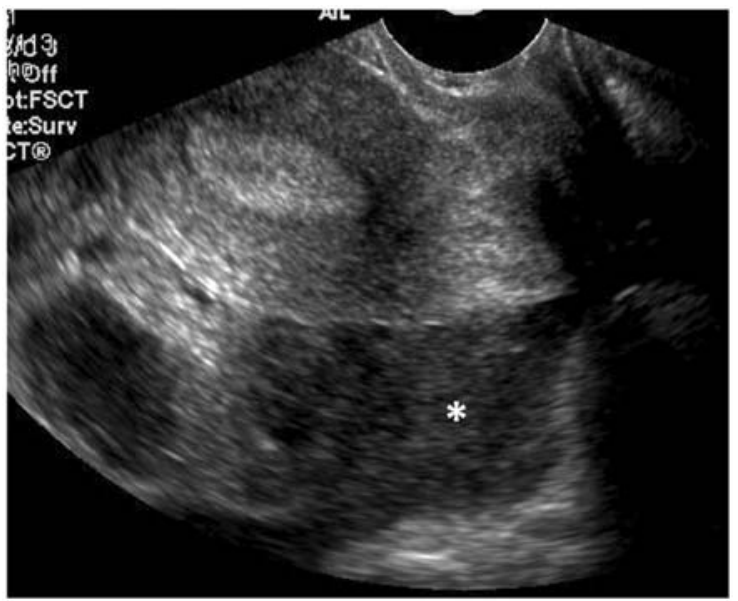

Figure 14 Complex haemorrhagic ascites $\left[^{\star}\right]$ in an ectopic pregnancy

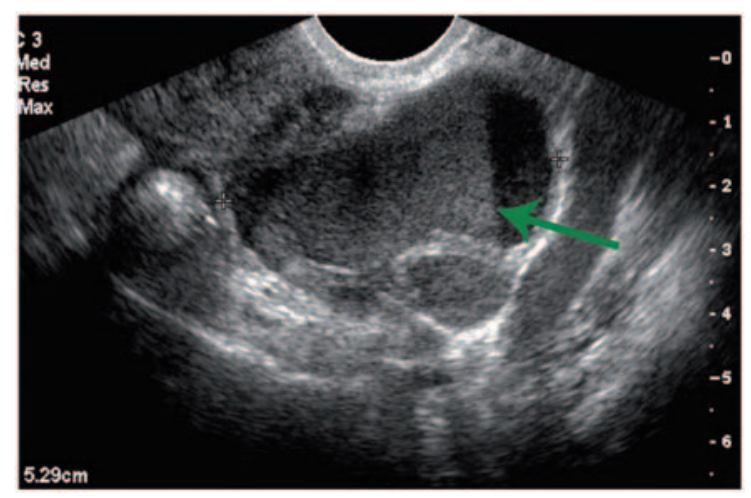

Figure 15 Fluid-fluid level [arrow] in a pyosalpinx

The sonographic findings are a convoluted tubular structure with an echogenic wall, representing the dilated tube; a fluid-fluid level representing pus or blood can sometimes be seen (Figure 15). The ovary should be visualised as a separate structure.

Dependent on the clinical situation, patients are often treated with intravenous antibiotics in the first instance, with surgery reserved for more complex cases or those that do not improve.

\section{Urological}

\section{Vesicoureteric junction stone}

Renal stone disease occurs in 5\% of the population, and in those who have previously presented with stone disease, the recurrence rate is $50 \% .^{9}$ Calculi can occur anywhere within the urinary tract.

The ureter is relatively narrow at three points: the pelviureteric junction (PUJ), where the ureter crosses the common iliac vessels and at the vesicoureteric junction (VUJ); and it is at these sites calculi are most likely to become lodged. If a partial or complete obstruction occurs, it can result in renal colic - acute and severe spasmodic flank pain, which can radiate to the groin or pelvic area.

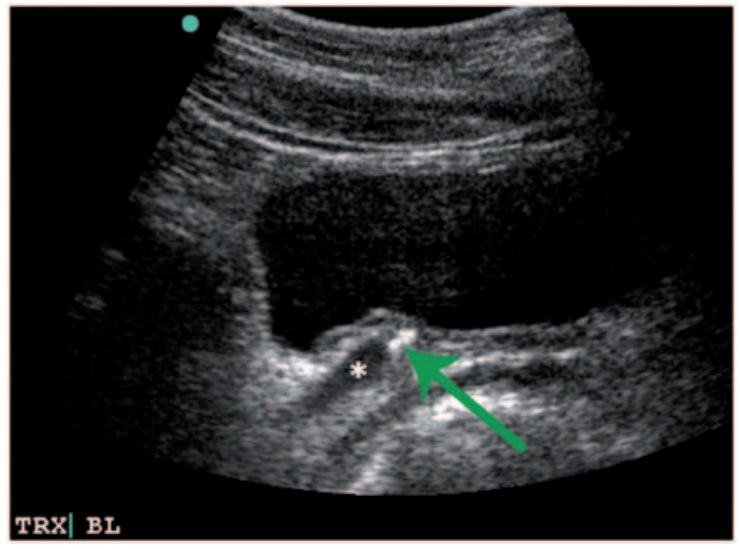

Figure 16 Well-defined echogenicity [arrow] representing a calculus at the VUJ with posterior acoustic shadowing $\left.{ }^{*}\right]$

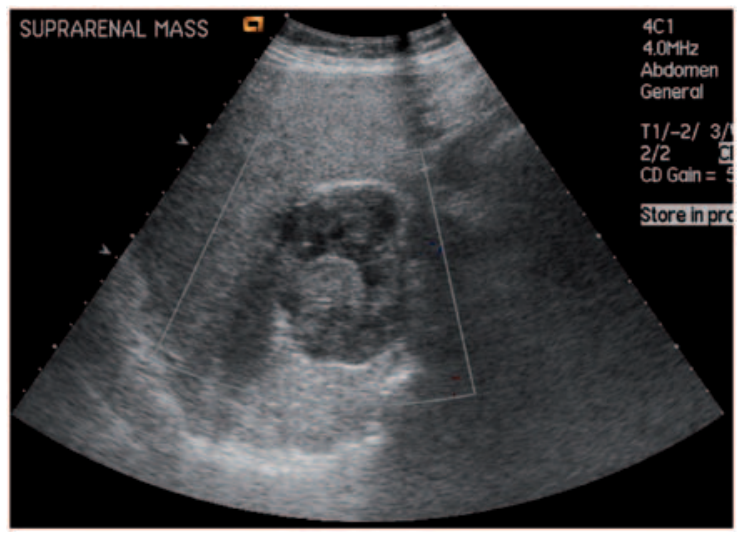

Figure 17 Complex echogenic mass representing adrenal haemorrhage

A non-contrast low-dose CT examination is now the gold standard investigation for the investigation of renal calculi. However, due to the variable presentation, ultrasound can often be the first investigation in these patients.

A calculus at the VUJ will be seen as a well-defined echogenicity with posterior acoustic shadowing (Figure 16). The ipsilateral ureteric jet, seen using colour Doppler, will be absent in cases of complete obstruction or reduced in partial obstruction. If there is resultant hydroureter and hydronephrosis, this can also be appreciated.

Management of a patient with VUJ calculi depends upon the size of the calculi (calculi less than $5 \mathrm{~mm}$ often pass spontaneously) and whether there is any hydronephrosis.

\section{Adrenal haemorrhage}

This is most commonly seen in neonates but can present in adults following trauma, acute physiological stress and in patients who are anticoagulated or who have a coagulopathy.

On ultrasound, a complex echogenic mass is seen superior to and separate from the upper pole of the kidney (Figure 17). The pelvis and paracolic region should be imaged for free fluid. The contralateral side should be carefully interrogated as in cases of bilateral haematomas, there is the potential for the development of adrenal 


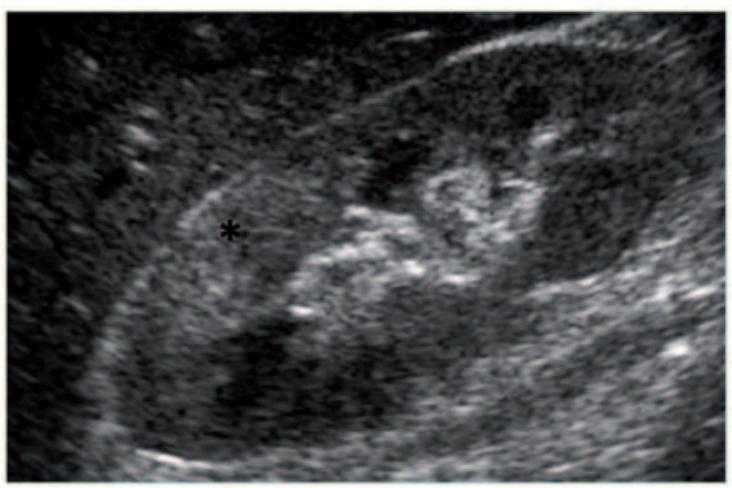

Figure 18 Focal area of increased echogenicity $\left[{ }^{*}\right]$ in a case of acute focal pyelonephritis

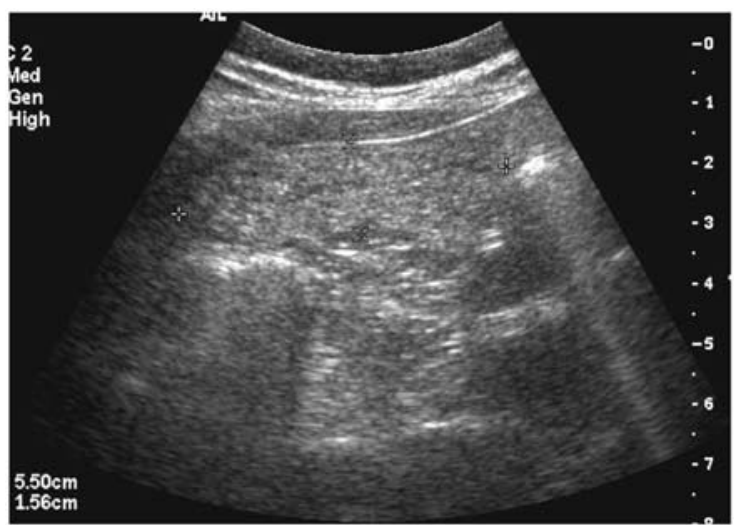

Figure 19 Omental infarction: focal area of increased echogenicity not related to solid abdominal organ

insufficiency. Diagnosis can be confirmed on CT imaging, which will reveal a high-density, enlarged adrenal gland.

Patients are often managed conservatively, as the adrenal haematoma usually involutes and resolves completely in a period of weeks to months. However, if the patient is haemodynamically unstable, secondary to persistent haemorrhage, procedures are usually required to stop the bleeding either with surgery or interventional radiology.

\section{Acute pyelonephritis}

Pyelonephritis is defined as an upper urinary tract infection with pelvic, calyceal and parenchymal inflammation. It is most commonly caused by initial colonisation with E. coli, which leads to paralysis of the ureteric smooth muscle function, culminating in dilatation and functional obstruction of the collecting system. Other potential pathways of infection include vesicoureteric reflux and haematogenous spread. Imaging indication is dependent on the patient's past medical history, as well as response to therapy and organism involved.

A variant of acute pyelonephritis is acute focal pyelonephritis. This involves single or multiple areas of renal inflammation. Patients affected by the focal form usually have a degree of immunosuppression, altered host resistance or have had trauma. ${ }^{8}$

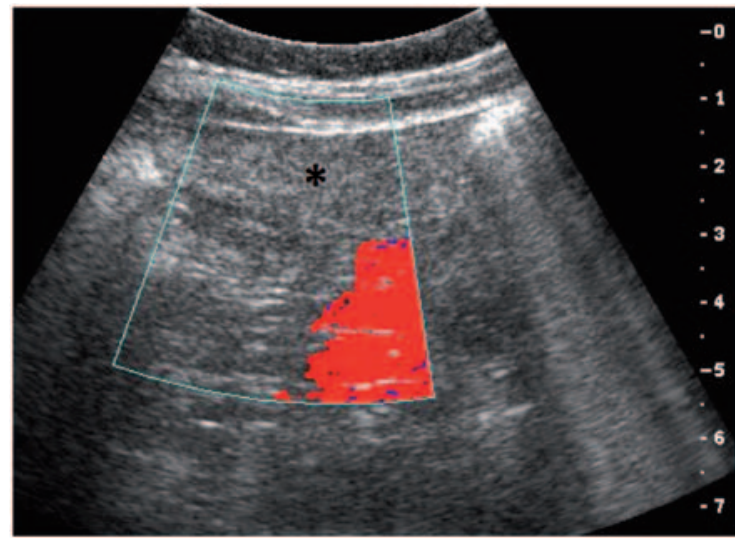

Figure 20 Absent Doppler flow within an area of omental infarction [ $\left.{ }^{\star}\right]$

Ultrasound is not a sensitive imaging modality for pyelonephritis with over $50 \%$ of scans having normal appearances. In the diffuse form, the kidney may be enlarged and appear hypoechoic, with surrounding hyperechoic fat due to inflammation. The corticomedullary differentiation may also be blurred. In focal pyelonephritis the findings are similar, but the areas of abnormal renal parenchyma are separated by normal parenchyma (Figure 18). Patients are treated conservatively with intravenous antibiotics. Careful inspection of the collecting system should be made to assess for pyonephrosis, which is seen as low-level echoes within the collecting system, as this may warrant drainage of the collecting system.

\section{Miscellaneous}

\section{Omental infarction}

This is a rare cause of acute abdominal pain that results from vascular compromise of a focal area of the greater omentum. This can either be primary or secondary in aetiology. In cases of primary omental infarction, the right iliac fossa is the most common site of pain. This is thought to be due to the more tenuous nature of blood supply of the right lateral free omental edge ${ }^{16}$ and the tendency of veins to kink deep within the anterior pelvis, particularly on the right side. ${ }^{17,18}$ It may also occur in hypercoagulable states. Secondary omental infarction may occur in trauma, surgical intervention or inflammation.

Sonographic findings include a well-defined focal area of increased echogenicity, which is not related to any solid abdominal organ (Figure 19) with absent Doppler flow within (Figure 20). The adjacent bowel is usually spared, although rarely a degree of wall thickening may be seen. ${ }^{16}$ A definitive diagnosis is difficult to make on clinical findings and ultrasound imaging alone, and further crosssectional imaging is usually required. Management is usually conservative, although it is important to be aware of potential complications such as abscess formation.

\section{Rectus sheath haematoma}

This is an uncommon and often misdiagnosed cause of abdominal pain due to its non-specific and varied presentation. It is the result of a direct tear to the rectus muscles or 


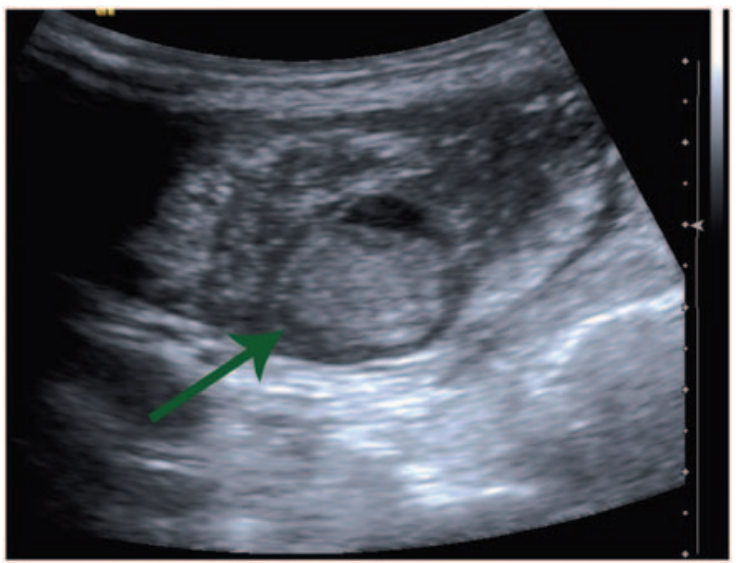

Figure 21 Well-defined echogenic area within the rectus muscle representing haematoma

following damage to the superior or inferior epigastric arteries or their branches. Spontaneous cases can occur in patients on anticoagulants when minor trauma can result in significant haematomas. $^{19}$

On clinical examination, Carnett's sign - unchanged or increase in abdominal pain upon tensing of the abdominal muscles - may be elicited, which points towards pathology within the abdominal wall rather than an intra-abdominal cause.

Sonographic appearances can be varied depending on the timing of the onset and the size of the haematoma. Most commonly, a well-defined echogenic area within the rectus muscle is seen (Figure 21). It is important to recognise the extraperitoneal location, which should help to direct towards the correct diagnosis and exclude other causes of abdominal pain.

Although usually self-limiting in nature, if there is sufficient expansion of the rectus sheath to accommodate a significant haematoma, hypovolaemic shock may develop, which requires appropriate resuscitation and occasionally vascular interventional radiology input. ${ }^{19}$ With the aging population and the increasing number of patients on anticoagulation therapy, it is an important diagnosis to consider in non-specific abdominal pain.

\section{Conclusion}

By following a systematic approach, it is possible to detect a number of sensitive sonographic findings that can aid diagnosis in the patient presenting with right iliac fossa pain. Ultrasound is a dynamic test which distinguishes it from other cross-sectional techniques and additionally it helps to limit exposure to ionising radiation, which is particularly important when managing conditions that commonly affect a young population.

\section{ACKNOWLEDGEMENTS}

We are grateful to the Radiology Department at Wythenshawe Hospital, UHSM for providing the images used in this article.

\section{DECLARATIONS}

Competing interests: None.

Funding: None.

Ethical approval: Not required

Guarantor: EKW

Contributorship: LM and VR conceived the article. LM, GJ and VR identified cases with appropriate images. EW wrote the first draft of the manuscript. EW, LM and VR reviewed and edited the manuscript and all authors approved the final version of the manuscript.

\section{REFERENCES}

1. Brant W, Helms C. Fundamentals of Diagnostic Radiology, 3rd edn. Philadelphia, PA: Lippincott Williams \& Wilkins, 2007

2. Rumack C, Wilson S, Charboneau JW. Diagnostic Ultrasound, 3rd edn. Philadelphia, PA: Mosby, 2004

3. Bendeck SE, Nino-Murcia M, Berry GJ, et al. Imaging for suspected appendicitis negative appendectomy and perforation rates. Radiology 2002;225:131-6

4. DiLaura S, Crum-Cianflone NF. Ileitis: When it is not Crohn's disease. Curr Gastroenterol Rep 2010;12:249-58

5. Karatepe $\mathrm{O}$, et al. Cecal diverticulitis mimicking acute appendicitis: a report of 4 cases. World J Emerg Surg 2008;3:16

6. Fenoglio-Preiser CM. Gastrointestinal Pathology an Atlas and Text. Philadelphia, PA: Lippincott Williams, 2008

7. Pradel JA, Adell cJF, Taourel P, et al. Acute colonic diverticulitis: prospective comparative evaluation with US and CT. Radiology 1997;205:503-12

8. Dahnert W. Radiology Review Manual, 7th edn. Baltimore, MD: Lippincott Williams \& Wilkins, 2011

9. Weissleder R, Wittenberg J, Harisinghani MG, et al. Primer of Diagnostic Imaging, 5th edn. Missouri: Mosby, 2011

10. del-Poso G, Albillos JC, Tejedor D, et al. Intussusception in children: current concepts in diagnosis and enema reduction. Radiographics 1999;19:299-319

11. Donnelly LF, Jones B, O'hara S, et al. Diagnostic Imaging - Pediatrics, 1st edn. Utah: Amirsys, 2005

12. Vijayaraghavan SB. Sonographic whirlpool sign in ovarian torsion. J Ultrasound Med 2004;23:1643-9

13. Okai T, Kobayashi K, Ryo E, et al. Transvaginal sonographic appearance of hemorrhagic functional ovarian cysts and their spontaneous regression. Int J Gynaecol Obstet 1994;44:47-52

14. Levine D, Brown DL, Andreotti RF, et al. Management of asymptomatic ovarian and other adnexal cysts imaged at US: Society of Radiologist in Ultrasound Consensus Conference Statement. Radiology 2010;256:943-54

15. Dogra V, Paspulati RM, Bhatt S. First trimester bleeding evaluation. Ultrasound $Q$ 2005;21:29-85

16. Kamaya A, Federle MP, Desser TS. Imaging manifestation of abdominal fat necrosis and its mimics. Radiographics 2011;31:2021-34

17. McClure MJ, Khalili K, Sarrazin J, et al. Radiological features of epiploic appendagitis and segmental omental infarction. Clin Radiol 2001;56:819-27

18. Singh AK, Alhilali LM, Gervais DA, et al. Omental infarct: an unusual CT appearance after superior mesenteric artery occlusion. Emerg Radiol 2004;10:276-8

19. Alia VM, Karnam SM, Kaushik M, et al. Spontaneous rectus sheath haematoma. West J Emerg Med 2010;11:76-9 